\title{
Prediction of gene expression from regulatory sequence composition enhances transcriptome-wide association studies
}

\author{
Federico Marotta ${ }^{1}$ Reza Mozafari ${ }^{1} \quad$ Elena Grassi ${ }^{2,3}$ \\ Alessandro Lussana ${ }^{4,5} \quad$ Elisa Mariella ${ }^{2,3}$ \\ Paolo Provero ${ }^{1,5, *}$ \\ ${ }^{1}$ Dept. of Neurosciences 'Rita Levi Montalcini', University of Turin, Italy \\ ${ }^{2}$ Dept. of Oncology, University of Turin, Italy \\ ${ }^{3}$ Fondazione del Piemonte per l'Oncologia, Istituto di Candiolo (IRCCS) Candiolo, Italy \\ ${ }^{4}$ Division of Immunology, Transplantation and Infectious Diseases, IRCCS San Raffaele \\ Scientific Institute, Milan, Italy \\ ${ }^{5}$ Center for Omics Sciences, Ospedale San Raffaele IRCCS, Milan, Italy \\ *corresponding author: paolo.provero@unito.it
}

\begin{abstract}
Transcriptome-wide association studies (TWAS) can prioritize trait-associated genes by finding correlations between a trait and the genetically regulated component of gene expression. A basic ingredient of a TWAS is a regression model, typically trained in an external reference data set, used to impute the geneticallyregulated expression. We devised a model that improves the accuracy of the imputation by using, as predictors, not the genotypes directly but rather the sequence composition of the proximal gene regulatory region, expressed as its profile of affinities for a set of position weight matrices. When trained on 48 tissues from GTEx, the regression model showed improved performance compared with models regressing expression directly on the genotype. We imputed the expression levels in genotyped individuals from the ADNI data set, and used the imputed expression to perform a TWAS. We also developed a method to perform the TWAS based on summary statistics from genome-wide association studies, and applied it to 11 complex traits from the UK Biobank. The greater accuracy in the prediction of gene expression allowed us to report hundreds of new gene-phenotype association candidates.
\end{abstract}




\section{Introduction}

Genome-wide association studies (GWAS) have revealed thousands of associations between genetic variants and complex traits or diseases [1, 2]. Most of such GWAS hits lie outside coding regions, and are likely to affect the phenotype by altering gene regulation, a view supported by various strands of evidence $[3,4,5]$. However the regulatory effects of a non-coding variant, and even its target genes, are difficult to predict. This fact, combined with linkage disequilibrium which makes it difficult to identify the truly causal variants among those showing statistically significant associations, makes the interpretation of GWAS hits and their eventual exploitation in devising new approaches to treatment extremely challenging.

Recently, new approaches have been developed to tackle these issues and, specifically, to derive gene/trait associations, rather than variant/trait associations, from GWAS data, based on intermediate molecular phenotypes. In these methods genetic variants are first associated, using a reference data set, to a suitable molecular phenotype such as mRNA expression [6, 7], splicing isoform abundance [8], or protein expression [9]. A predictive model is generated from the reference data and used to impute the intermediate molecular phenotype in the individuals assayed by the GWAS, then associations are sought between the imputed molecular phenotype and the trait of interest.

Based on the putative regulatory effect of GWAS hits, gene expression is a natural choice of intermediate phenotype, and was indeed used in the first such studies, which were thus called transcriptome-wide association studies (TWAS) [6]. To train the predictive model of gene expression, TWAS can rely on large dataset, such as GTEx [10], where genotypes and gene expression data in various tissues are available for hundreds of individuals. TWAS can also be perfomed when only GWAS summary statistics are available, by combining in a statistically appropriate way the expression weights with the GWAS Z-scores [7, 11].

As the final goal of a TWAS is the discovery of associations between a phenotype and the genetically-regulated component of gene expression, a reliable method to infer the genetically-regulated component of expression is needed. The common way to tackle this problem is to build, for each gene, a regression model where the outcome is the expression and the predictors are the $0 / 1 / 2$-encoded genotypes for all the markers falling in a predefined window around the gene $[6,12]$. Since the predictive model uses only DNA-derived features as predictors, the differences in expression that it captures represent the genetically-determined variance in gene expression.

Since the seminal paper by Gamazon et al. [6], most predictive models applied to TWAS have used, as predictors, a direct $0 / 1 / 2$ encoding of the genotypes, and the differences between the tools proposed so far amount to the level of sophistication of the statistical learning model employed. For instance, PrediXcan [6] uses elastic net; FUSION [7] relies on an ensemble of models, including a best linear unbiased predictor (BLUP) and a Bayesian sparse linear mixed model (BSLMM); and TIGAR [13] introduces a complex hierarchical Bayesian model. Of notice, HAPLEXER [14] is different in that it uses signature tract sets (i.e. sets of haplotypes shared between individuals) instead of genotypes, and TF2Exp [15] uses the predicted differences in the binding of 
transcription factors to their target sites.

In this work, we present a new strategy, whereby gene expression is predicted from the sequence composition of a gene's proximal regulatory region. Sequence composition is usually expressed as the frequency spectrum of k-mers; here, based on our previous work on eQTLs [16], we chose to parametrize sequence composition as the spectrum of total bindig affinity (TBA) for a set of position weight matrices (PWMs) associated to transcription factors (TFs), as these are the DNA motifs most likely to impact gene expression. A gene's regulatory sequence is thus represented by a TBA profile, which is used as regressor in predicting the variation of gene expression across individuals.

We show that this strategy outperforms the previously proposed ones, even using a relatively simple regression model such as Ridge regression, and even though we consider only proximal regulatory regions as opposed to large windows around each gene. We also describe how our strategy can be used when only GWAS summary statistics are available, albeit in an approximate form. Our models were trained in 46 tissues and 2 cell lines from GTEx (in the following collectively denoted as "tissues") and applied to perform genotype-level TWAS for 43 Alzheimer's-related phenotypes measured in the Alzheimer Disease Neuroimaging Initiative (ADNI), and summarylevel studies for 11 complex traits in the UK Biobank, allowing us to report hundreds of new genes potentially associated to complex traits or diseases. We also developed and made available a convenient suite of scripts to run these analysis (Section 4.11).

\section{Results}

\subsection{Prediction of gene expression from TBA profiles}

In our approach variation across individuals of the expression of a gene in a given tissue is modeled as depending on the TBA for a set of PWMs of the proximal regulatory region of the gene. The TBA, inspired by a statistical-mechanical model [17], integrates the signal given by the PWM along all the possible binding sites in the regulatory region. We have previously shown that it can be used to effectively encode differences in gene regulation among genes [18], species [19], and individuals [16]. Therefore, our model assumes that genetically determined differences among individuals in the expression of a gene in a given tissue are mediated by the differences in the TBA profiles of the gene's regulatory region.

For each gene, we defined its regulatory region as the union of all the sequences extending from 1500 base pairs (bps) upstream to 500 bps downstream of the transcription start site (TSS) of all the annotated transcripts; then, given a library of PWMs and a reference panel of individuals with their genomes sequenced, we can compute the TBA for each PWM of the regulatory region of each gene in each individual. The TBA values of the reference individuals can then be used, instead of the genotypes, as independent variables in a penalized regression model where the dependent variable is gene expression. Preliminary analysis suggested that Ridge regression outperformed both least absolute shrinkage and selection operator (Lasso) and elastic net in predicting expression, thus Ridge regression was used to build all the predictive 
models. The genetically regulated component of expression has been previously abbreviated with GReX [6]; since our model finds the genetic component mediated by the total binding affinity, we called our tool TReX, from "TBA-Regulated eXpression." We also refer to the imputed expression as $\widehat{T R e} X$.

Using the subset of white individuals from the GTEx data set [10] as a reference panel and Hocomoco [20] for the position weight matrices, we computed the affinity for 640 PWMs and trained our model to predict the expression of both coding and noncoding genes in 48 tissues. The number of genes in each tissue was close to 40,000, whereas the number of individuals ranged from 70 for substantia nigra to 421 for skeletal muscle. We thus trained almost two million models to predict the expression of a gene in a given tissue starting from the DNA sequence of its regulatory region in a given individual.

As it is customary $[6,13,15]$, in order to assess the predictive performance of our Ridge model andcompare it with that of other tools, we used 5-fold cross-validation, assessing the performance by the $R^{2}$ value, defined as the squared correlation coefficient between true and predicted expression values in the validation fold, averaged over the 5 folds. We compared the cross-validation $R^{2}$ achieved by TReX with those achieved on the same data by TIGAR [13], which, to the best of our knowledge, is currently the best performing prediction tool based on the same performance metric and the same data.

Table 1 shows some statistics related to the distribution of 5-fold cross-validated $R^{2}$ s obtained with the two tools in EBV-transformed lymphocytes (Fig. 1 (a and b)) and brain cortex (Suppl. Fig. 1). The difference in performance was statistically significant $\left(P<2.2 e-16\right.$, paired Wilcoxon test on $R^{2}$, in both tissues). Cross-validated $R^{2}$ values for all GTEx tissues are reported in Suppl. Tab. 1. The improvement in performance brought by TReX was significant in both protein coding and non-coding genes considered separately, but the increase in cross-validated $R^{2}$ was significantly higher for protein-coding genes $(P<2.2 e-16$ in both tissues; Mann-Whitney $\mathrm{U}$ test $)$.

The improvement in predictive performance was not limited to cross-validated samples but extended to the prediction of gene expression in samples from a different dataset and population: When we used our model to predict gene expression in LCLs in a sample of 89 Yoruba individuals from the Geuvadis dataset, we obtained again $R^{2}$ values significantly higher that those obtained with TIGAR (Fig. 1 (c) and Table 1). About half the genes with $R^{2}>0.1$ according to TIGAR also have $R^{2}>0.1$ according to TReX (Suppl. Fig. 2), suggesting that the genes on which the two methods are most effective do not coincide, so that integrating the two could provide even greater predictive power.

A direct comparison with TF2Exp [15], which like TReX predicts expression variation based on predicted differences in transcription factor affinity, is made problematic by the fact that TF2Exp was trained on the Geuvadis LCL dataset rather than of GTEx tissues. Qualitatively, both the percentage of genes with cross-validated $R^{2}>0.05(21911 / 40056=54.7 \%)$ and the mean $R^{2}(0.0709)$ of TReX are higher than the corresponding values reported [15] for TF2Exp (respectively 20.1\% and 0.049). More generally, TF2exp uses cell-type specific epigenetic information which is not readily available for most of the GTEx tissues (see also Discussion). 
bioRxiv preprint doi: https://doi.org/10.1101/2021.05.11.443571; this version posted May 26, 2021. The copyright holder for this preprint

a

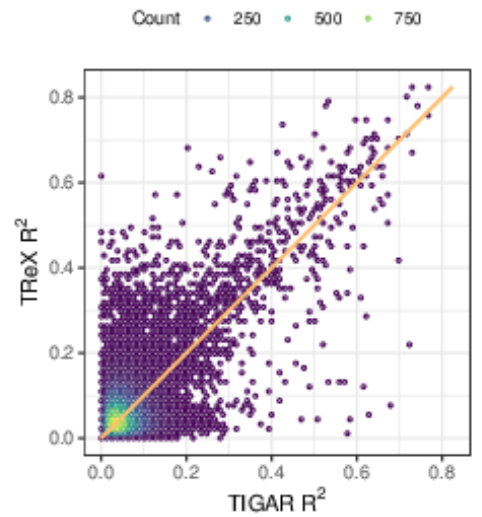

b

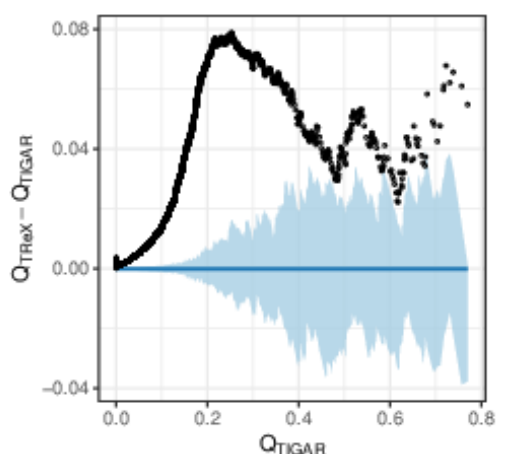

C

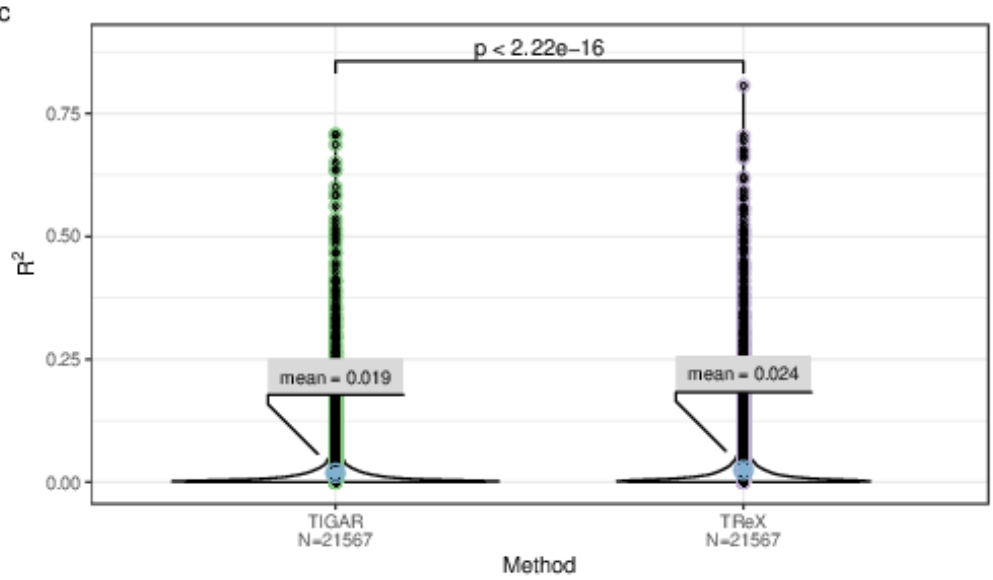

Figure 1: Predictive performance of various models. a. Scatter plot of the 5 -fold crossvalidation $R^{2}$ for TIGAR and TReX on EBV-transformed lymphocytes; the plot shows 39905 genes. In principle each point represents a gene, but in order to reduce overplotting we associated potentially many genes that fell approximately in the same region to a single point; the color of a point denotes the number of genes associated to it. The orange line is the identity line. b. Detrended QQ-plot of the 5-fold cross-validation $R^{2}$ for TIGAR and TReX on EBV-transformed lymphocytes. The $\mathrm{x}$ axis represents the quantiles of TIGAR's $R^{2}$, while on the y axis there are the differences between corresponding quantiles from TReX's and TIGAR's $R^{2}$. The confidence interval (light blue) was generated from 100 bootstrap replicates from the distribution of TIGAR's $R^{2}$. c. Predictive performance in an external data set. Distribution of the squared correlation between the true and predicted expression values in 89 Yoruban individuals from the GEUVADIS data set (21567 genes common to both methods). 


\begin{tabular}{l|rr|rr|rr} 
& \multicolumn{2}{|c|}{ GTEx LCL } & \multicolumn{2}{|c|}{ GTEx brain cortex } & \multicolumn{2}{r}{ Youruban LCL } \\
\hline & TReX & TIGAR & TReX & TIGAR & TReX & TIGAR \\
\hline median $R^{2}$ & 0.0546 & 0.0496 & 0.0448 & 0.0409 & 0.0089 & 0.0071 \\
mean $R^{2}$ & 0.0709 & 0.0623 & 0.0641 & 0.0575 & 0.0244 & 0.0187 \\
\# genes & 39905 & 39905 & 39770 & 39770 & 21567 & 21567 \\
\# genes with $R^{2}>0.01$ & 38896 & 38605 & 38030 & 37875 & 10199 & 9180 \\
\# genes with $R^{2}>0.05$ & 21911 & 19802 & 17526 & 15512 & 2759 & 1913 \\
\# genes with $R^{2}>0.1$ & 7419 & 5616 & 5650 & 4285 & 993 & 570
\end{tabular}

Table 1: $R^{2}$ distribution for two GTEx tissues and an external dataset representing a different ethnicity. $R^{2}$ is defined as the square of the correlation between predicted and actual values (averaged over 5 cross-validation folds in the GTEx tissues).

Therefore encoding the genotype as a profile of total binding affinities for PWMs led to a significant improvement of the prediction, even if only genetic variants within a region of length $\sim 2,000$ were used to compute such profiles. It would be tempting to interpret these results as reflecting the underlying biology of gene regulation, by stating that the TBA profiles capture the genetic variation that is responsible for interindividual differences in TF binding, which is in turn responsible for differences in gene expression. However, models trained with scrambled versions of the position weight matrices, which are unlikely to reflect the binding preferences of any TF, gave results very similar to those obtained with the original PWMs (Suppl. Fig. 3). Therefore, the reason for the improvement in performance must be that affinity profiles for PWMs provide an efficient encoding of sequence composition allowing non-linear effects of the genotype on expression to be taken into account (see also Discussion).

\subsection{Genotype-level TWAS}

We computed the total binding affinities of the Hocomoco PWMs for the proximal regulatory regions of all the genes in 735 white individuals from the ADNI data set for which the genotypes and 43 Alzheimer's disease (AD)-related phenotypes were available. Then, using the models previously trained on the 48 GTEx tissues, we imputed the expression in these individuals from their binding affinities, and found associations between the predicted expression and each of the phenotypes using linear or logistic regression, according to whether the phenotype was quantitative or categorical. In all regressions we included the same set of covariates (see section 4.6). Two of the phenotypes were categorical and pertained to the diagnosis at the baseline visit, while the 41 quantitative phenotypes included volumetric measures of brain regions, quantifications of biomarkers such as TAU protein and amyloid $\beta$, and results of cognitive tests such as the Preclinical Alzheimer Cognitive Composite (PACC), the Alzheimer's Disease Assessment Scale-Cognitive (ADAS), or the Rey's Auditory Verbal Learning Test (RAVLT). The associations were investigated on a gene-by-gene basis, therefore, in total, we tested about 10 million associations. Given the high correlation among the 
phenotypes considered and among the predicted gene expression in different tissues, we implemented an independent Bonferroni correction for each tissue/phenotype pair, finding 179 significant associations involving 84 genes, 47 tissues, and 43 phenotypes (Suppl. Table 2 and Section 4.12). The strongest associations (Figure 2 c, d) appeared to be biologically meaningful and supported by the scientific literature. As it will be shown in the next section, we exploited this TWAS on ADNI also to compare the results of the genotype- and summary-level TWAS on the same data.

The strongest association with disease status among protein-coding genes was shown by the expression of CAPN15 in the colon transverse. CAPN15 was also the top scoring gene for the association with disease status in seven other tissues (hypothalamus, esophagus mucosa, ovary, pancreas, prostate, skin - not sun exposed suprapubic, skin - sun exposed lower leg; Figure 2 c). The calpains, to which this gene belongs, are a family of cysteine proteases whose hyperactivation has been previously related to Alzheimer's disease [21]; furthermore, a recent preprint describes how the knockout of CAPN15 in mice impairs brain development and plasticity [22]. Interestingly, CAPN15 has not been previously associated to AD through a GWAS according to the EBI GWAS catalog, showing that our method can find new gene/disease associations.

PPP5D1, a protein-serine/threonine phosphatases, was also strongly associated to disease status in multiple tissues. This gene is poorly characterized in the literature, but in the GWAS catalog it is associated to late-onset AD. INTS9 likely plays a role in small nuclear RNA processing [23], and was found differentially expressed in AD patients [24]. Remarkably, we also found NECAB3 among the top-scoring genes: This calcium binding protein inhibits the association of X11L with amyloid precursor protein and abolishes the suppression of beta-amyloid production by X11L [25, 26]. We could not find any reliable reference describing an association between TMIGD1 and Alzheimer's disease, but it was associated with Crohn's disease [27], and in turn inflammatory bowel disease seems to be related to Alzheimer's [28].

To further demonstrate our results on the ADNI data set, we chose a particular tissue, the hypothalamus, and plotted both the GWAS and the TWAS for disease status together (Figure 2 a). Notably, many TWAS hits do not correspond to a GWAS peak.

The results for the quantitative phenotypes were analyzed together by finding, for each tissue, the gene with the strongest association considering all 41 phenotypes together, thus obtaining a list of one gene per tissue. The top 10 genes by maximum absolute Z-score together with the number of tissues in which they show the strongest association are shown in Figure $2 \mathbf{d}$.

The genetic component of MAF1 expression in three tissues (brain cortex, cervical spine, and gastroesophageal junction) is strongly and negatively associated to the volume of the hippocampus; consistently, it was recently reported that MAF1 inhibits dendritic morphogenesis and the growth of dendritic spines, and affects learning and memory in mice [29]. Notably, the association between MAF1 expression in the hippocampus itself and hippocampal volume was not significant. This apparent contradiction could be explained either by an effect of the phenotype on gene expression (which, for continuous phenotypes, would be present also in the reference dataset; see Supplementary Note, Sec.1.2) or by a genetic variant pleiotropically affecting both 
bioRxiv preprint doi: https://doi.org/10.1101/2021.05.11.443571; this version posted May 26, 2021. The copyright holder for this preprint

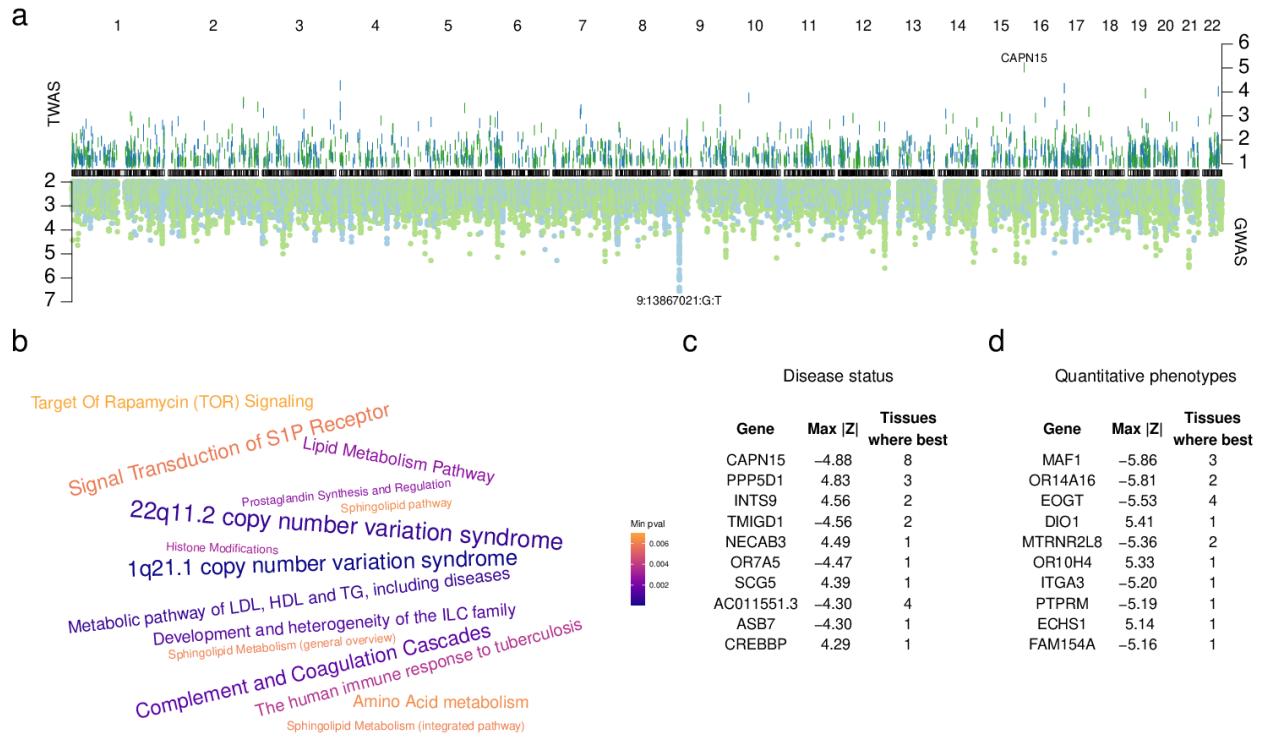

Figure 2: Transcriptome-wide association study on the ADNI data set. a. Double Manhattan plot for disease status. The Z-scores of allgenetic markers (bottom panel) and all genes with cross-validated $R^{2}>0.1$ (top panel) are shown as a function of their genomic location. For the TWAS, the Ridge model for the imputation of gene expression was trained on the hypothalamus GTEx data. Blue (green) points or bars indicate positive (negative) association with disease status encoded as 0 for controls and 1 for patients diagnosed as Alzheimer's disease, early/late mild cognitive impairment, or significant memory concern (see 4.5). b. Top enriched pathways for the TWAS on the "Ecog" quantitative phenotype. The size of each term is proportional to the number of tissues in which the term is enriched with an adjusted p-value smaller than 0.05, ranging from 1, (e.g. Histone Modifications), to 7 (22q11.2 copy number variation syndrome). The color of each term represents the smallest adjusted enrichment p-value. c, d. Top genes associated to disease status or a quantitative phenotype. Associations between the disease status and the predicted expressions were found through logistic regression; disease status was encoded in two different ways as described in 4.5. Associations with quantitative phenotypes were assessed through linear regression. The tables report, for each gene, the maximum Z-score across all 48 tissues, and the number of tissues where that gene had the best Z-score for at least one of the phenotypes. 
expression and phenotype.

Also the imputed expression of EOGT significantly correlates with several Alzheimerrelated phenotypes, although this gene has not been implicated in AD by GWAS studies. The role of EOGT is to attach sugar moieties to extracellularly-secreted or membrane proteins [30]. Glycosylation is known to regulate the cleavage of the amyloid precursor protein (APP) [31], and has also been implicated in many forms of neurodegeneration [32, 33].

To the best of our knowledge, all attempts to find associations between polymorphisims in the DIO1 gene (whose product, D1, can activate or inactivate the thyroid hormone) and cognitive functions so far have failed [34, 35], despite the fact that thyroid function is linked to Alzheimer's disease [36]. Here, we discovered a positive, relatively strong association between the $T \widehat{\operatorname{Re} X}$ of DIO1 in the adrenal gland and the results of the EcogSPDivatt test.

So far we have only reported associations with protein-coding genes, but we actually found many associations with pseudogenes or other non-coding genes. These associations are more difficult to explain because the genes involved are not well-documented in the literature.

We also investigated whether relevant biological pathways are enriched among the top-scoring genes. For each tissue-phenotype pair, we selected the genes with Z-score greater than 3 in absolute value, and analyzed them for enriched pathways from the WikiPathways database. After pooling together all the four phenotypes pertaining to RAVLT scores, we considered the pathways with an enrichment adjusted p-value smaller than 0.05 , and counted in how many different tissue each pathway appeared; the results are shown in Figure 2 b.

In the literature, we could find evidence for the implication of all these terms to cognitive disorders. For instance, 22q11.2 CNV syndrome is known to alter the structure of the brain [37] and lead to cognitive decline [38]. Another CNV syndrome, 1q21.1, was detected in Alzheimer's disease patients [39]. Inflammation has been long known to lead to several diseases, including neurodegenerative ones; thus, it is not surprising here to find pathways related to innate lymphoid cells (ILC), complement and coagulation [40,41]. We also found many terms related to lipids, which is, again, not surprising, as lipids play many fundamental roles in the brain: from the processing of amyloid precursor protein (APP) to receptor signaling, from myelination to membrane remodeling, from oxidation to energy balance; the implication of lipids in Alzheimer's disease has been reported many times [42, 43, 44].

In conclusion, although multiple testing correction was performed separately for each tissue/phenotype pair, inspection of the significant associations seems to confirm the validity of the approach. To exploit GWAS data derived from much greater samples, but available only as summary results, we developed a strategy to perform a summarylevel TWAS using our affinity-based weights, and applied it to the UK Biobank, where summary GWAS results based on hundreds of thousand of individuals are available. 


\subsection{Summary-level TWAS}

As we mentioned in the previous section, the sample size in data sets where the genotype of each individual is available is still very limited, either for privacy concerns or logistic reasons [7]. Much more commonly, only the summary statistics of a GWAS are available; Gusev et al. [7] developed a methos to perform a TWAS using only a model trained in a reference data set and the GWAS summary statistics. However, their method was developed to work with the 0/1/2-encoded genotypes rather than with genotypes summarized as TBA profiles or otherwise. Here, we extended their method so that it could be applied also when the expression-predicting model is trained on TBA profiles (see section 4.9). Briefly, we computed the change in TBA determined by each SNP, and combined it with the weights learned during the training phase to find the change in expression determined by each SNP through the induced changes TBA. Thus, we reduced to the original case and could apply the association test developed by Gusev et al. in an approximate version. We performed a summary-level TWAS on the same ADNI data used for the genotype-level TWAS described above, and we found the results of summary-leval and genotype-level TBA-based TWAS to be highly correlated (Figure 3 a).

We thus decided to perform a summary-level TWAS on 11 phenotypes from the UK Biobank, including height, body mass index, bone mineral density, platelet count, and various other blood-related metrics (Suppl. Table 3). We used 1e-9 as the study-wide significance threshold for the p-value; this number accounts for testing about 40,000 genes in 48 tissues for 11 phenotypes.

To determine whether TReX has an advantage over other existing tools, we trained the models for FUSION on the same brain cortex data as TReX, and compared the results. Overall, the Z-scores by FUSION and TReX are quite correlated (Pearson corr. 0.38 for the Z-scores pertaining to the TWAS on height, Suppl. Fig. 4). However, TReX found a considerable number of genes with p-value $<1 \mathrm{e}-9$ that were not significant for FUSION. In the height TWAS, TReX detected even more significant genes than FUSION (Figure 3 b).

The complete lists of significant associations between genes and the 11 UK Biobank phenotypes analyzed is available as a supplementary file (Section 4.12). Here we discuss some notable examples. By far, the phenotype where we could detect the greatest number of associations was height, with 3526 unique genes having an association pvalue smaller than 1e-9 in at least one tissue, and each association detected on average in about 13 tissues. Moreover, 166 genes showed a p-value smaller than 1e-9 in all 48 tissues. At the opposite end, red blood cell count has the smallest number of associations, with only 125 unique genes showing p-values smaller than the threshold (Table 2).

UQCC1 is well-known for its association with human height [45]; consistently, we found that the expression of this gene in the frontal cortex of the brain is strongly and negatively associated with height. Similarly, ATOH7 and GH2, both previously reported in the GWAS catalog, were also found to be strongly associated with height in our TWAS. At the same time, we found genes that were not reported in the GWAS catalog, but whose association was supported by functional evidence. One example is 
bioRxiv preprint doi: https://doi.org/10.1101/2021.05.11.443571; this version posted May 26, 2021. The copyright holder for this preprint

a

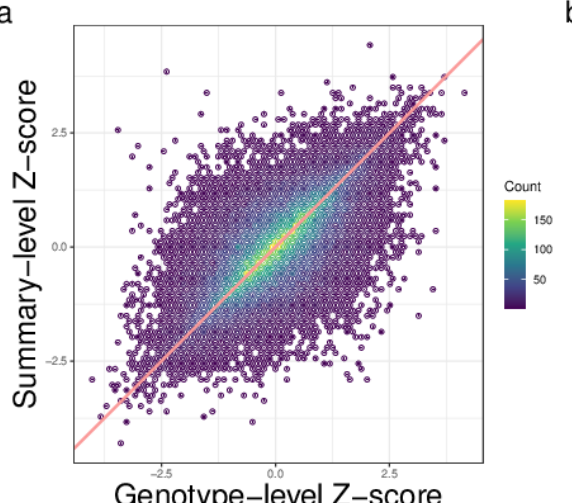

$b_{\text {Fusion }}$

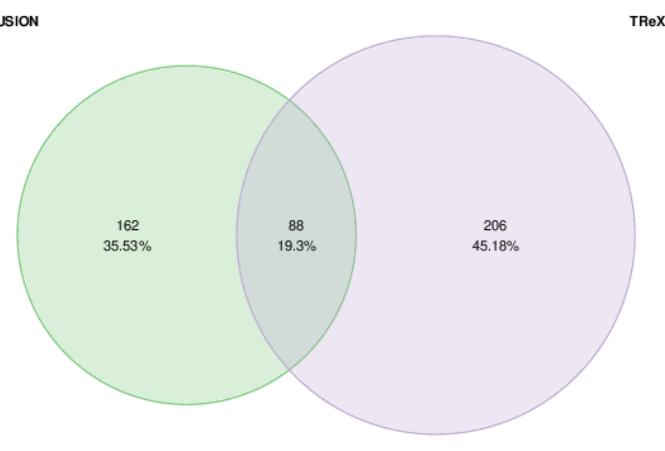

C

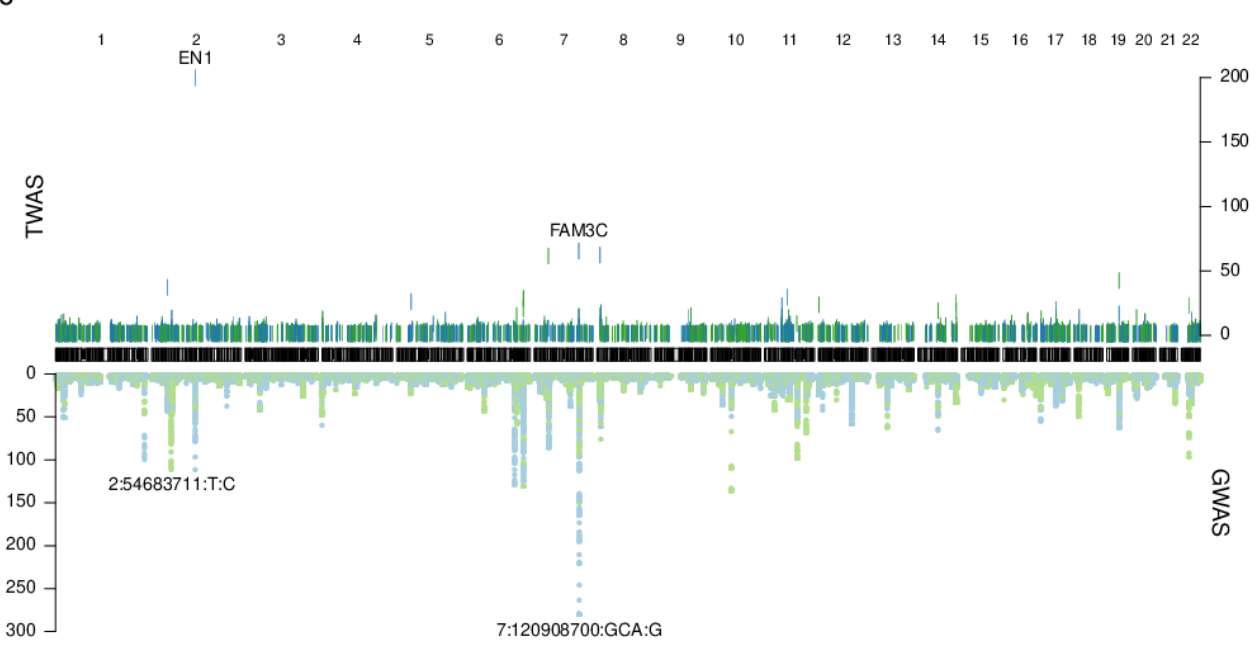

Figure 3: Summary-level TWAS. a. Comparison of the genotype- and summary- level TWAS for TReX. In both cases the weights were learned in the brain cortex of GTEx white samples and the phenotypes was the level of amyloid $\beta$ at the baseline visit for white ADNI samples. For the summary-level TWAS, the linkage disequilibrium matrix was obtained from all GTEx white samples. Pearson correlation: $0.66, \mathrm{t}=166.47$, $\mathrm{df}=36012$, $\mathrm{p}$-value $<2.2 \mathrm{e}-16$. b. Comparison of genes found by FUSION and TReX. The Euler diagram shows the protein-coding genes with an association p-value smaller than 1e9. The weights were trained in 120 white individuals from GTEx using the expression values from the brain cortex, and the Z-scores are from the height GWAS. c. Double Manhattan plot for BMD. The top panel shows the genes whose predicted expression in brain cortex is associated with the bone mineral density; the bottom panel shows the SNPs associated to this trait in the UK Biobank data set. 


\begin{tabular}{lccc}
\hline Phenotype & $\begin{array}{c}\text { Genes in the } \\
\text { TReX TWAS }\end{array}$ & $\begin{array}{c}\text { Genes in the } \\
\text { GWAS catalog }\end{array}$ & $\begin{array}{c}\text { Genes in } \\
\text { common }\end{array}$ \\
\hline height & 3526 & 3469 & 712 \\
platelet count & 2107 & 1282 & 341 \\
eosinophil count & 1454 & 1170 & 239 \\
RBC distr width & 1287 & 1145 & 211 \\
WBC count & 1138 & 1374 & 179 \\
BMI & 1067 & 119 & 25 \\
FVC & 1065 & 732 & 71 \\
FEV1 & 1033 & 114 & 20 \\
BMD & 748 & 72 & 12 \\
mean corpuscular haemoglobin & 502 & 1328 & 56 \\
RBC count & 124 & 1429 & 3 \\
\hline
\end{tabular}

Table 2: Number of significant genes for each phenotype. We performed a summarylevel TWAS on 11 phenotypes from the UK Biobank; the table reports, for each phenotype, the number of genes with a p-value smaller than 1e-9 in at least one tissue. The threshold comes from $0.05 /(40,000 \times 48 \times 11)$, since we tested the association of about 40,000 genes in 48 tissues for 11 phenotypes. The third column reports the number of genes reported by the GWAS catalog for the phenotype.

FRMD8, which promotes growth factor signaling [46]: its expression in the hypothalamus was positively associated with height. IPP is another promising candidate for further investigations, as its expression is negatively correlated with height; this gene, according to STRING [47], interacts with KLHL28, which in turn is known to be associated with body height.

Bone mineral density (Figure $3 \mathbf{c}$ and Suppl. Table 4) was associated with 645 genes, among which EN1 (a homeobox-containing gene) is prominent in that it is the most strongly associated gene to any of the 11 phenotypes. Conditional loss of EN1 is associated with low bone mass in mice [48], and many rare variants within this gene have also been previously associated to bone density [48, 49]. Strikingly, the expression of TP53INP2 is associated to bone density in all 48 GTEx tissues, but it is not reported in the GWAS catalog. This gene promotes autophagy and can act as a transcriptional activator, but we found no mention of its involvement with bone mineral density in the literature.

\section{Discussion}

We developed a new way to predict the genetic component of the expression of a gene from the sequence composition of its proximal regulatory sequence parametrized by its profile of affinities for position weight matrices. The profile of affinities of a regulatory region provides an efficient non-linear encoding of the genotype which allows 
the regression model to capture a larger fraction of the expression variance compared with regression models directly using the SNP genotypes as their independent variables. Contrary to our initial expectations, the predictive power of TBA profiles is not directly linked to their ability to predict transcription factor binding [18], as scrambled PWMs, which do not represent the binding preferences of actual transcription factors, led to very similar results. Therefore, the tranformation of genotype data into TBA profiles should rather be interpreted as a process of feature expansion, i.e. the creation of new features from non-linear combinations of the original features: Indeed the number of PWMs we use to encode the regulatory regions (640) is much higher than the typical number of variants in the regions we consider (mean 11.79 variants).

A related method to predict the variation of expression across individuals was recently put forward by Shi and collaborators [15], who used the DeepSEA [50] algorithm trained on a lymphoblastoid cell line to predict the effect of variants on the binding of transcription factors to their target sites (identified by ChIP-seq on the same cell line). Such predicted binding changes are then used as independent variables in regularized regression to develop predictive models of gene expression. An important difference with our method is that TF2Exp uses cell-type specific epigenetic information (by using DeepSEA trained on lymphoblastoid cell lines, and ChIP-seq and HiC data derived from the same cells to identify binding sites and assigning them to genes). Therefore our method, in which gene expression in the training data is the only tissue-specific information used, is more readily usable in a variety of tissues for which epigenetic information is not yet available. On the other hand, the relationship between variants and transcription factor binding is much more explicit in TF2Exp, which might thus better capture the causal relationship between alterations of TF binding and gene expression.

It is surprising that our model achieves such good results by just considering $\sim 2,000$ bps of proximal regulatory regions, although also variant-based methods such as TIGAR, while considering in principle SNPs in much larger regions, show larger effect sizes for those in the proximity of the TSS, and the same was observed for eQTLs [51]. Limiting the analysis to proximal regions leads to a decrease in both signal and noise, in such a way that their ratio might be actually increased. It should also be kept in mind that the proximal sequences do contain, through linkage disequilibrium, significant information about genetic variants located tens of thousands of base pairs away. Finally, proximal regulatory regions can be assigned to their target genes with much more confidence than distal ones, thus reducing the risk of multiple-hit genes (that is, multiple genes being associated to a phenotype based on the same locus [52]). When we tried to include enhancers in our model we faced two problems, the first being how to associate the enhancers to their target genes in a tissue-specific way, and the second being how to combine the TBAs of proximal and distal regions. As our attempts, so far, did not result in a significant increase in cross-validated $R^{2}$ compared with the proximal-only models used here, solving these two problems will be the focus of our next efforts.

Our method is not immune to the general limitations of the TWAS approach, including the inability to distinguish causation from pleiotropy, hence we would still recommend that TWAS be complemented by other methods such as colocalisation 
and mendelian randomization.

In conclusion, regressing gene expression on regulatory sequence composition parametrized thorugh PWMs leads to improved prediction of the genetic component of gene expression, thus allowing the discovery of new gene/trait associations via TWAS.

\section{Methods}

\subsection{Genotypes}

We obtained individual-level genotypes from three large-scale projects: GTEx v7 [53] (accession number phs000424.v7.p2), GEUVADIS [54], and ADNI (http://adni. loni.usc.edu/). Our method requires phased haplotypes, but not all the VCF files provided by the three above projects were phased. For GTEx, we performed the phasing using Beagle (28Sep18.793) with the HapMap GrCh37 genetic map. For GEUVADIS, the haplotypes were already phased for most of the samples and we discarded those for which they were not. For ADNI, we performed the phasing and imputation using Beagle (18May20.d20) [55, 56], using the 1000 Genomes Project phase 3 as the reference panel and the HapMap GrCh37 genetic map (everything was downloaded from http://faculty. washington.edu/browning/beagle/beagle. html), setting the window to $70.0 \mathrm{cM}$; the conform-gt program was used beforehand to adjust the positions and allele order of the genetic variants of the raw VCF to match the reference panel, as suggested in Beagle's manual.

The individual-level, phased genotypic data obtained from the three projects were uniformly processed before performing further analyses. For each data set, the VCF files were filtered using plink2 [57] as follows (the command line options are indicated in brackets). First, the files were separated by population (--keep); for this work we focused on white individuals for GTEx, white individuals for ADNI, and Yoruban individuals for GEUVADIS. We removed the genetic variants that failed QC tests (--var-filter) or that were duplicated (--rm-dup). Individuals with more than $10 \%$ of the markers missing were excluded (--geno 0.1). Variants with a Hardy-Weinberg p-value (with the "midp" correction) less than $10^{-10}$ were filtered out (--hwe $1 \mathrm{e}-10$ midp). Only biallelic markers (--max-alleles 2) with a minor allele frequency of at least $1 \%(--\operatorname{maf} 0.01)$ were retained. Moreover, the variant names were set to a unique, unambiguous identifier (--set-all-var-ids ' $@$ :\#:\$r:\$a' --new-id-max-allele-len 100 missing). Finally, the result was exported again in VCF format (--export id-delim=',' vcf-4.2)

\subsection{Total binding affinity}

In a 2006 article by Foat et al. [17], a statistical-mechanical model was used to derive a formula for the occupancy of a DNA sequence by a transcription factor, motivated by the fact that in many experiments (such as ChIP-chip or differential mRNA expression) the signal is, at least approximately, proportional to the occupancy. Inspired by this work, Molineris et al. [19], introduced a similar approach, but with two novelties: (i) 
PWMs are used to compute the affinity, and (ii) for each possible binding site, the score is given by the maximum between the forward and reverse strand. In particular, the total binding affinity of the transcription factor associated to the PWM $m$ for the regulatory region of gene $g$ is defined as

$$
\begin{aligned}
T B A_{g} & =\sum_{i=1}^{L_{g}-L_{m}+1} \max \left(S_{g}^{(+)}(i), S_{g}^{(-)}(i)\right), \\
S_{g}^{(+)}(i) & =\prod_{j=1}^{L_{m}} \frac{m(j, g(i+j-1))}{b(g(i+j-1))}, \\
S_{g}^{(-)}(i) & =\prod_{j=1}^{L_{m}} \frac{m\left(L_{m}-j+1, g^{\prime}(i+j-1)\right)}{b(g(i+j-1))},
\end{aligned}
$$

where: $S_{g}^{(+)}(i)$ and $S_{g}^{(-)}(i)$ are the scores of motif $m$ starting at position $i$ of sequence $g$ on the forward and reverse strands, respectively; $g(i)$ is the base at position $i$ of the regulatory region $g ; g^{\prime}(i)$ is the base at the same position but on the opposite strand, i.e. the Crick-Watson complement of $g(i) ; m(i, g(i))$ is the probability of observing base $g(i)$ at position $i$ in the PWM of transcription factor $k ; b(g(i))$ is the background probability of observing base $g(i)$.

The computation of the total binding affinity requires the genotypes in VCF format, the reference DNA sequence of the model organism, a BED file specifying the coordinates of the regulatory regions of each gene, the PWMs of all the transcription factors, and the background nucleotide frequencies. Helper scripts to find the regulatory regions and compute the total binding affinity are provided with this article (Section 4.11). In particular, the script to compute the total binding affinity relies on a specialized program called vcf_rider [58]. For this article, the affinity was computed for white individuals in GTEx [10], white individuals in ADNI, and Yoruban individuals in GEUVADIS [54], using the UCSC hg19 sequence as reference.

The regulatory regions associated to a gene were chosen to be sequences spanning $(-1500,+500)$ from the transcription start site of all annotated transcripts of the gene. The annotation of the transcripts was derived as follows. For GTEx and ADNI, we used the reference annotation from the GTEx portal (https://gtexportal.org/ home/datasets, downloaded on 07/25/2019), whereas for GEUVADIS we used GENCODE v12 [59]; these annotations contain the coordinates of the transcripts whose expression was measured. If the regulatory regions of two or more transcripts associated to the same gene overlapped, they were merged. After computing the total binding affinity for the set of (non-overlapping) regulatory regions of all the transcripts of a gene, they were summed and $\log _{2}$-transformed.

The PWMs were obtained from Hocomoco 10 [20]: we downloaded the mononucleotide models for humans. The background frequencies were calculated on the intergenic regions of the hg19 reference human sequence. 


\subsection{Gene expression}

Gene expression data in the form of RPKMs and read counts were obtained from the GTEx [53] and GEUVADIS [54] projects, and were preprocessed as reported in the article describing the analysis of GTEx v7 [53]. Briefly, we kept only the genes with RPKM $>0.1$ and read count $>5$ in at least 10 samples, we applied a quantile normalization across genes and an inverse-normal transformation across samples, and finally we subtracted the effect of covariates such as the PEER factors [60] (more details below).

Quantile normalization is used to minimize non-biological differences between individuals with respect to the RNA-sequencing [61]. Using the jargon of [61], each individual is treated as a dataset and each gene as an observation. The observed quantiles are projected onto the unit diagonal, and after this operation the distribution of gene expression is the same for all the individuals. An inverse-normal transformation [62] is then applied so that the distribution of the expression of each gene across individuals becomes standard normal. These transformations are applied to expression data for all tissues together, then the expression data are split by tissue.

Before training the models for the TReX imputation expression data were replaced by their residuals after the linear regression EXPRESSION $\sim$ COVARIATES. The covariates for each tissue were downloaded from the GTEx portal and included: sex, sequencing platform, the first three principal components (PCs) of the genotype, and a variable number of PEER factors depending on the number of samples with available expression for that tissue. The PEER factors were computed on the top 10000 genes and their number was $\min (15, \operatorname{Ceiling}($ SampleSize/5)) if the sample size was $<150$, 30 if the sample size was $\geq 150$ and $<250,35$ otherwise.

Although the GEUVADIS expression data had already been preprocessed by the authors of the original study, we started from the raw RPKM and read counts and appiled the above procedure also on the GEUVADIS data so as to be able to compare the results across the GTEx and GEUVADIS data sets.

\subsection{TReX imputation}

In the classical approach, where each SNP is encoded as 0,1 , or 2 according to its genotype, the following model is fitted for each gene:

$$
Y_{g}=\omega_{g, 0}+\sum_{j=1}^{m} \omega_{g, j} X_{g, j}+\epsilon_{g}
$$

Here, $Y_{g}$ is the residualized expression of gene $g, m$ is the total number of markers in a window (tipically $1 \mathrm{Mbp}$ ) around gene $g$, and $X_{g, j}$ is the genotype at locus $j$. The intercept $\omega_{g, 0}$ captures the baseline expression value for gene $g$, while the error term $\epsilon$ captures the non-cis-genetic variance of gene expression. Typically, both the expression and the genotypes are centered and scaled, so that the incercept can be ignored.

In our approach, the effect of many SNPs is integrated over the length of the proximal regulatory region of gene $g$ through the total binding affinity. The total binding affinity 
of each PWM captures the effect of all the SNPs simultaneously, although each SNP will have different effects on different PWMs according to which motifs it breaks or creates. Our Ridge regression fits the following model for each gene,

$$
Y_{g}=\beta_{g, 0}+\sum_{k=1}^{l} A_{g, k} \beta_{g, k}+\epsilon_{g},
$$

where $l$ is the total number of available PWMs ( 640 for HOCOMOCO v10), and $A_{g, j}$ is the $\log _{2}$-transformed total binding affinity for transcription factor $k$, as outlined in 4.2 .

Model performance was evaluated for all tissues by 5 -fold nested cross validation, whereby the samples are split in 5 groups and the model is trained 5 times, each time leaving one of the groups out and using the samples in that group to evaluate the performance of the model trained on all the other samples. Furthermore, in order to estimate the best value of the Ridge penalty factor, $\lambda$, we used a nested 10 -fold cross-validation, where each training group is further split in 10 subfolds and each subfold is used in turn to evaluate the performance of a model trained by choosing the parameter from a grid of possible values; this inner cross-validation loop is used to find the optimal value of the parameter $\lambda$ in a way that does not bias the evaluation of the performance that is carried out in the outer loop. For the sake of obtaining the prediction weigths, however, the outer cross-validation loop was omitted and only the inner, 10-fold loop was retained.

The results of the training are $l$ weights for each gene, where each weight represent the effect of the affinity of one particular PWM on the expression of that gene. Then, it is possible to compute the total binding affinities of the promoters of each gene in a testing data set for all the individuals, and to use the weights to "predict" the expression values in the testing data set. Software for performing all these tasks is avalable (see Section 4.11).

\subsection{Phenotypes}

For the ADNI data set, we started from the adnimerge table provided by the ADNIMERGE R package version 1 (https://adni.bitbucket.io/), which contained various phenotypes measured at multiple time points for each individual, during the baseline visit and subsequent follow ups. We kept only individuals annotated as "white" and for which the genotypes were available, resulting in 735 samples, and for them we retained only the values maesured at the baseline visit. Furthermore, we excluded the following phenotypes because we used them as covariates: age (at baseline), sex, years of education, ethnicity, marital status, number of APOE epsilon4 alleles, intracranial volume (at baseline).

The disease status at baseline was originally encoded as a categorical variable with the following values: healthy controls $(\mathrm{n}=253)$, early mild cognitive impairment ( $\mathrm{n}$ $=211)$, late mild cognitive impairment $(\mathrm{n}=228)$, significant memory concern $(\mathrm{n}=$ $0)$, Alzheimer's disease $(n=43)$. We recoded this variable in two different ways and considered the resulting variables as two distinct phenotypes: in the first, AD samples 
were encoded as " 1 " and all the others as " 0 ", whereas in the second CN samples were encoded as " 0 " and all the others as "1". We found that the latter encoding provided the most significant associations, possibly because the two classes are of similar size, whereas in the first encoding, only $5 \%$ of the individuals belong to the " 1 " class. However, when discussing associations with the disease status in the results, we refer to associations with any of these two encodings.

All the other 41 remaining baseline phenotypes were quantitative; examples of these include volumetric measures of various regions of the brain, scores of cognitive tests, and quantifications of amyloid beta and tau proteins. Quantitative phenotypes that ranged outside of the sensitivity intervals (and were therefore denoted like " $<x$ " or " $>x ")$ were truncated to $x$.

\subsection{Genotype-level TWAS}

Association between the $\widehat{T R e} X$ in each tissue and each phenotype was assessed by linear or logistic regression for quantitative and binary phenotypes, respectively. For all the regressions, the formula was $Y \sim \widehat{T R e} X+C O V A R I A T E S$, where $\widehat{T R e} X$ is the imputed expression and the covariates are: age (at baseline), gender, years of education, ethnicity, marital status, number of APOE epsilon4 alleles, intracranial volume (at baseline), and the first ten principal components of the genotype. Aside from the PCs of the genotype, which were computed with plink2 from the genotypes preprocessed as described in section 4.1, all the other covariates were derived from the adnimerge table from the ADNIMERGE $\mathrm{R}$ package. The Z-score and the p-value of the association between expression and phenotype were defined as, respectively, the standardised coefficient and the p-value of the variable $\widehat{T R e} X$ in the regression model. All the software required to perform the genotype-level TWAS is publicly available (Section 4.11).

\subsection{GWAS}

In order to compare the genotype-level with the summary-level TWAS we needed the summary statistics of a GWAS performed on the ADNI data set. We used plink2 to convert the population-specific VCF files preprocessed as described in section 4.1 into plink's binary format, and passed to plink2 the phenotypes and covariates processed as described in section 4.5. Besides --glm and the options for the genotpe, phenotype, and covariates files, the only additional option we passed to plink2 was --covar-variance-standardize.

\subsection{Summary statistics}

For the ADNI data set, the output of plink2 consisted of the summary statistics; for the UK Biobank, we downloaded summary statistics for 11 of the 7221 phenotypes which were considered as part of a GWAS analysis across 6 continental ancestry groups by the Neale lab [63]. We processed all the summary statistics first with fizi munge and then with fizi impute (https://github.com/bogdanlab/fizi). The former parses 
the summary statistics and performs some quality control checks before printing the data in a standardized format, while the latter imputes the missing Z-scores.

fizi can leverage functional data to improve the imputation, but in this case we did not use this feature, and performed the imputation using only a reference linkage disequilibrium panel. The reference linkage disequilibrium file is simply a plink . bed file with the genotypes of all the individuals in a data set. Here, we derived the reference LD file from the GTEx data set processed as described in section 4.1, thus including only white individuals. During the imputation procedure, a variant present in the reference file but without a Z-score is assigned a Z-score computed from the scores of its neighbouring variants and the respective linkage disequilibrium in the reference panel.

\subsection{Summary-level TWAS}

In the original approach to a summary-level TWAS [7], the association test relies on the following Wald statistic:

$$
Z_{t}=\frac{\mathbf{W Z}_{g}}{\sqrt{\mathbf{W} \boldsymbol{\Sigma}_{g, g} \mathbf{W}^{T}}},
$$

where: $Z_{t}$ is the TWAS Z-score; $\mathbf{Z}_{g}$ is the vector of GWAS Z-scores of the SNPs within $1 \mathrm{Mbp}$ around the gene; $\mathbf{W}$ is the vector of weights, one for each SNP, obtained by the predictive model; and $\boldsymbol{\Sigma}_{g, g}$ is the linkage disequilibrium matrix between the SNPs in the window. In practice, $\boldsymbol{\Sigma}_{g, g}$ is unknown because we lack the full genotypes from the GWAS data set, but it can be estimated from a separate reference linkage disequilibrium panel.

This approach requires in particular $\mathbf{W}$, the effect of each SNP on gene expression, while we only have the effect of the affinity on gene expression. However, since the total binding affinity is a deterministic function of the genotype (section 4.2), we can compute the approximate change in total binding affinity caused by each SNP, and from that the change in gene expression caused by each SNP. We computed this quantity empirically as the difference between the mean affinity of all the individuals with genotype 0 and the mean affinity of all the individuals with genotype 1 in a reference data set. Since we had already computed the affinities for all the individuals in GTEx, we used it as the reference for computing what we call the $\Delta_{T B A}$.

For this article we used GWAS Z-scores either from the GWAS that we performed on ADNI (4.7) or from the UK Biobank; we preprocessed them as described in section 4.8. Summary results for $11 \mathrm{UK}$ Biobank complex traits were obtained from the Neale Lab web site (http://www.nealelab.is/uk-biobank) and are listed in Suppl. Table 3. The expression weights $\beta$ were trained on white individuals in GTEx, and both the $\Delta_{T B A}$ and $\boldsymbol{\Sigma}$ were derived from the genotypes of white individuals in GTEx. All the scripts are available on GitHub (Section 4.11). 


\subsection{TIGAR and FUSION}

We downloaded the software from GitHub (https://github.com/yanglab-emory/ TIGAR and https://github.com/gusevlab/fusion_twas) and followed the authors' instructions to compute the weights using our own genotype and expression files (the same we used for TReX). For TIGAR, we trained the DPR model and evaluated the 5 -fold cross-validation $R^{2}$. For FUSION we trained all the available models except BSLMM due to the high computational cost of the latter; in all cases we used the default parameters specified by the authors of the software.

\subsection{URLs}

GitHub repository https://github.com/fmarotta/TReX

\subsection{Supplementary files}

\section{- TWAS_ADNI_significant.tsv}

- TWAS_UKBB_significant.tsv

\subsection{Acknowledgements}

The Genotype-Tissue Expression (GTEx) Project was supported by the Common Fund of the Office of the Director of the National Institutes of Health (commonfund.nih.gov/GTEx). Additional funds were provided by the NCI, NHGRI, NHLBI, NIDA, NIMH, and NINDS. Donors were enrolled at Biospecimen Source Sites funded by NCI Leidos Biomedical Research, Inc. subcontracts to the National Disease Research Interchange (10XS170), Roswell Park Cancer Institute (10XS171), and Science Care, Inc. (X10S172). The Laboratory, Data Analysis, and Coordinating Center (LDACC) was funded through a contract (HHSN268201000029C) to the The Broad Institute, Inc. Biorepository operations were funded through a Leidos Biomedical Research, Inc. subcontract to Van Andel Research Institute (10ST1035). Additional data repository and project management were provided by Leidos Biomedical Research, Inc.(HHSN261200800001E). The Brain Bank was supported supplements to University of Miami grant DA006227. Statistical Methods development grants were made to the University of Geneva (MH090941 \& MH101814), the University of Chicago (MH090951,MH090937, MH101825, \& MH101820), the University of North Carolina - Chapel Hill (MH090936), North Carolina State University (MH101819),Harvard University (MH090948), Stanford University (MH101782), Washington University (MH101810), and to the University of Pennsylvania (MH101822). The datasets used for the analyses described in this manuscript were obtained from dbGaP at http://www.ncbi.nlm.nih.gov/gap through dbGaP accession number phs000424.v7.p2/GRU. 


\section{References}

[1] Peter M Visscher et al. "10 years of GWAS discovery: biology, function, and translation." In: Am J Hum Genet 101.1 (July 2017), pp. 5-22. ISSN: 00029297. DOI: $10.1016 / \mathrm{j}$.ajhg.2017.06.005. URL: http : / / linkinghub. elsevier. com/retrieve/pii/S0002929717302409 (visited on 01/01/2019).

[2] Vivian Tam et al. "Benefits and limitations of genome-wide association studies." In: Nat Rev Genet 20.8 (2019), pp. 467-484. ISSN: 1471-0056. DOI: 10 . 1038 / s41576-019-0127-1. URL: http: //www . nature.com/articles/s41576-0190127-1 (visited on 12/02/2020).

[3] Matthew T Maurano et al. "Systematic localization of common disease-associated variation in regulatory DNA." In: Science 337.6099 (Sept. 2012), pp. 1190-1195. DOI: $10.1126 /$ science.1222794. URL: http://dx.doi.org/10.1126/science. 1222794 (visited on 12/02/2020).

[4] Claudia Giambartolomei et al. "Bayesian test for colocalisation between pairs of genetic association studies using summary statistics." In: PLoS Genet 10.5 (May 2014), e1004383. DOI: 10 . $1371 /$ journal . pgen . 1004383. URL: http : //dx.doi.org/10.1371/journal.pgen.1004383 (visited on 12/02/2020).

[5] Kyle Kai-How Farh et al. "Genetic and epigenetic fine mapping of causal autoimmune disease variants." In: Nature 518.7539 (Feb. 2015), pp. 337-343. DOI: 10. 1038 / nature13835. URL: http:// dx . doi . org/10 . 1038 / nature13835 (visited on $01 / 13 / 2015$ ).

[6] Eric R Gamazon et al. "A gene-based association method for mapping traits using reference transcriptome data." In: Nat Genet 47.9 (Sept. 2015), pp. 10911098. DOI: $10.1038 / \mathrm{ng}$. 3367. URL: http://dx.doi .org/10.1038/ng . 3367 (visited on $12 / 11 / 2018$ ).

[7] Alexander Gusev et al. "Integrative approaches for large-scale transcriptomewide association studies." In: Nat Genet 48.3 (Mar. 2016), pp. 245-252. DoI: 10.1038/ng. 3506. URL: http://dx.doi .org/10.1038/ng. 3506 (visited on $06 / 16 / 2020)$.

[8] Alexander Gusev et al. "A transcriptome-wide association study of high grade serous epithelial ovarian cancer identifies novel susceptibility genes and splice variants". In: Nat Genet 51.5 (May 2019), pp. 815-823. ISSN: 1061-4036. DOI: 10.1038/s41588-019-0395-x. URL: https : //www.ncbi.nlm.nih.gov/pmc/ articles/PMC6548545/ (visited on 03/25/2021).

[9] Nadav Brandes, Nathan Linial, and Michal Linial. "PWAS: proteome-wide association study-linking genes and phenotypes by functional variation in proteins." In: Genome Biol 21.1 (July 2020), p. 173. DOI: 10.1186/s13059-020-02089x. URL: http : / / dx . doi . org/10.1186/s13059-020-02089-x (visited on 12/02/2020). 
[10] François Aguet et al. "Genetic effects on gene expression across human tissues". en. In: Nature 550.7675 (Oct. 2017). Number: 7675 Publisher: Nature Publishing Group, pp. 204-213. ISSN: 1476-4687. DOI: 10.1038/nature24277. URL: https: //www. nature.com/articles/nature24277 (visited on 03/25/2021).

[11] Alvaro N Barbeira et al. "Integrating predicted transcriptome from multiple tissues improves association detection." In: PLoS Genet 15.1 (Jan. 2019), e1007889. DOI: 10 .1371/journal .pgen . 1007889. URL: http : //dx . doi .org/10 . 1371/ journal.pgen.1007889 (visited on 12/02/2020).

[12] Ohad Manor and Eran Segal. "Robust prediction of expression differences among human individuals using only genotype information." In: PLoS Genet 9.3 (Mar. 2013), e1003396. DOI: 10.1371/journal.pgen.1003396. URL: http://dx.doi. org/10.1371/journal.pgen.1003396 (visited on 12/02/2020).

[13] Sini Nagpal et al. "TIGAR: an improved bayesian tool for transcriptomic data imputation enhances gene mapping of complex traits." In: Am J Hum Genet 105.2 (Aug. 2019), pp. 258-266. ISSN: 00029297. DOI: 10.1016/j .ajhg.2019.05. 018. URL: https://linkinghub.elsevier.com/retrieve/pii/S0002929719302058 (visited on $07 / 03 / 2019$ ).

[14] Berk A Alpay et al. "Combinatorial and statistical prediction of gene expression from haplotype sequence." In: Bioinformatics 36.Supplement_1 (July 2020), pp. i194-i202. DOI: 10.1093/bioinformatics/btaa318. URL: http://dx.doi . org/10.1093/bioinformatics/btaa318 (visited on 07/21/2020).

[15] Wenqiang Shi, Oriol Fornes, and Wyeth W Wasserman. "Gene expression models based on transcription factor binding events confer insight into functional cisregulatory variants." In: Bioinformatics 35.15 (Aug. 2019), pp. 2610-2617. DOI: 10 . 1093 / bioinformatics / bty992. URL: http : / / dx . doi . org / 10 . 1093 / bioinformatics/bty992 (visited on 12/28/2020).

[16] Elena Grassi et al. "A functional strategy to characterize expression Quantitative Trait Loci." In: Hum Genet 136.11-12 (Nov. 2017), pp. 1477-1487. DOI: 10.1007/ s00439-017-1849-9. URL: http://dx.doi.org/10.1007/s00439-017-1849-9 (visited on 05/04/2020).

[17] Barrett C Foat, Alexandre V Morozov, and Harmen J Bussemaker. "Statistical mechanical modeling of genome-wide transcription factor occupancy data by MatrixREDUCE." In: Bioinformatics 22.14 (July 2006), e141-9. DOI: 10.1093/ bioinformatics/bt1223. URL: http://dx.doi.org/10.1093/bioinformatics/ bt1223 (visited on 12/28/2020).

[18] Elena Grassi et al. "Total binding affinity profiles of regulatory regions predict transcription factor binding and gene expression in human cells." In: PLoS ONE 10.11 (Nov. 2015), e0143627. DOI: 10.1371/journal . pone.0143627. URL: http: //dx.doi.org/10.1371/journal.pone.0143627 (visited on 09/11/2020). 
[19] Ivan Molineris et al. "Evolution of promoter affinity for transcription factors in the human lineage." In: Mol Biol Evol 28.8 (Aug. 2011), pp. 2173-2183. DoI: 10.1093/molbev/msr027. URL: http://dx.doi.org/10.1093/molbev/msr027 (visited on 12/28/2020).

[20] Ivan V. Kulakovskiy et al. "HOCOMOCO: expansion and enhancement of the collection of transcription factor binding sites models". In: Nucleic Acids Research 44.D1 (Jan. 2016), pp. D116-D125. ISSN: 0305-1048. DOI: 10.1093/nar/gkv1249. URL: https://doi.org/10.1093/nar/gkv1249 (visited on 03/26/2021).

[21] Yacoubou Abdoul Razak Mahaman et al. "Involvement of calpain in the neuropathogenesis of Alzheimer's disease". In: 39.2 (Mar. 2019), pp. 608-630. ISSN: 10981128. DOI: 10 . 1002 / med . 21534. URL: /pmc/articles / PMC6585958 / ?report=abstract \%20https : / / www . ncbi . nlm . nih . gov / pmc/articles / PMC6585958/.

[22] Congyao Zha et al. "Disruption of capn15 in mice leads to brain and eye deficits". In: (Mar. 2019), p. 763888. DOI: 10.1101/763888. URL: https://doi.org/10. $1101 / 763888$.

[23] David Baillat et al. "Integrator, a multiprotein mediator of small nuclear RNA processing, associates with the C-terminal repeat of RNA polymerase II". eng. In: Cell 123.2 (Oct. 2005), pp. 265-276. ISSN: 0092-8674. DOI: 10.1016/j.cell. 2005.08 .019 .

[24] Diego Mastroeni et al. "Laser-captured microglia in the Alzheimer's and Parkinson's brain reveal unique regional expression profiles and suggest a potential role for hepatitis B in the Alzheimer's brain". In: Neurobiol. Aging 63 (Mar. 2018), pp. 12-21. ISSN: 15581497. DOI: $10.1016 /$ j. neurobiolaging . 2017 . 10 . 019. URL: /pmc/articles/PMC6686891/?report=abstract\%20https : / www . ncbi . nlm.nih.gov/pmc/articles/PMC6686891/.

[25] D. S. Lee et al. "Regulation of X11L-dependent amyloid precursor protein metabolism by XB51, a novel X11L-binding protein". eng. In: J Biol Chem 275.30 (July 2000), pp. 23134-23138. ISSN: 0021-9258. DOI: $10.1074 /$ jbc.C000302200.

[26] Akio Sumioka et al. "XB51 isoforms mediate Alzheimer's $\beta$-amyloid peptide production by X11L (X11-like protein)-dependent and -independent mechanisms". In: Biochem. J. 374.1 (2003), pp. 261-268. ISSN: 02646021. DOI: 10 . 1042 / BJ20030489. URL: http://www.genome.ad.jp.

[27] Yamile Zabana et al. "Transcriptomic identification of TMIGD1 and its relationship with the ileal epithelial cell differentiation in Crohn's disease". In: Am. J. Physiol. - Gastrointest. Liver Physiol. 319.2 (Aug. 2020), G109-G120. ISSN: 15221547. DOI: 10.1152/ajpgi.00027.2020. URL: https://pubmed.ncbi.nlm. nih.gov/32508154/.

[28] Bing Zhang et al. "Inflammatory bowel disease is associated with higher dementia risk: A nationwide longitudinal study". In: Gut 0 (2020), pp. 1-7. ISSN: 14683288. DOI: 10 . 1136/gutjnl-2020-320789. URL: http : / / dx . doi . org/10 . 1136/ gutjnl-2020-320789. 
[29] Kui Chen et al. "Maf1 regulates dendritic morphogenesis and influences learning and memory". In: Cell Death Dis. 11.7 (July 2020). ISSN: 20414889. DOI: 10 . 1038/s41419-020-02809-y.

[30] Mitsutaka Ogawa, Koichi Furukawa, and Tetsuya Okajima. "Extracellular Olinked $\beta$-N-acetylglucosamine: Its biology and relationship to human disease". In: World J Biol Chem 5.2 (May 2014), pp. 224-230. ISSN: 1949-8454. DOI: 10. 4331/wjbc.v5.i2.224. URL: https://www.ncbi.nlm.nih.gov/pmc/articles/ PMC4050115/ (visited on 04/27/2021).

[31] Peng Fei Tao and Han Chang Huang. "Regulation of A $\beta$ PP Glycosylation Modification and Roles of Glycosylation on A $\beta$ PP Cleavage in Alzheimer's Disease". In: ACS Chem. Neurosci. 10.5 (May 2019), pp. 2115-2124. ISSN: 19487193. DOI: 10. 1021 /acschemneuro.8b00574.

[32] Tobias Moll, Pamela J Shaw, and Johnathan Cooper-Knock. "Disrupted glycosylation of lipids and proteins is a cause of neurodegeneration". In: Brain 143.5 (May 2020), pp. 1332-1340. ISSN: 14602156. DOI: 10.1093/brain/awz358.

[33] Wagner B. Dias and Gerald W. Hart. "O-GlcNAc modification in diabetes and Alzheimer's disease". In: Mol. Biosyst. 3.11 (2007), pp. 766-772. ISSN: 17422051. DOI: $10.1039 / \mathrm{b} 704905 f$.

[34] Herman Verloop et al. "Genetics in endocrinology: Genetic variation in deiodinases: A systematic review of potential clinical effects in humans". In: Eur. J. Endocrinol. 171.3 (Sept. 2014), R123-R135. ISSN: 1479683X. DOI: 10.1530/EJE-14-0302.

[35] Frank Jan De Jong et al. "The association of polymorphisms in the type 1 and 2 deiodinase genes with circulating thyroid hormone parameters and atrophy of the medial temporal lobe". In: J. Clin. Endocrinol. Metab. 92.2 (Feb. 2007), pp. 636-640. ISSN: 0021972X. DOI: 10.1210/jc.2006-1331.

[36] Patrick Quinlan et al. "Altered thyroid hormone profile in patients with Alzheimer's disease". In: Psychoneuroendocrinology 121 (Nov. 2020). ISSN: 18733360. DOI: 10.1016/j.psyneuen.2020.104844.

[37] David R. Roalf et al. "White matter microstructural deficits in 22q11.2 deletion syndrome". In: Psychiatry Res. - Neuroimaging 268 (Oct. 2017), pp. 35-44. ISSN: 18727506. DOI: 10.1016/j.pscychresns.2017.08.001.

[38] Jacob A.S. Vorstman et al. "Cognitive decline preceding the onset of psychosis in patients with 22q11.2 deletion syndrome". In: JAMA Psychiatry 72.4 (Apr. 2015), pp. 377-385. ISSN: 2168622X. DOI: 10.1001/jamapsychiatry. 2014. 2671.

[39] Denis Cuccaro et al. "Copy number variants in Alzheimer's disease". In: J. Alzheimer's Dis. 55.1 (Nov. 2016), pp. 37-52. ISSN: 18758908. DOI: 10.3233/JAD-160469.

[40] Franca Orsini et al. "Versatility of the complement system in neuroinflammation, neurodegeneration and brain homeostasis". In: Front. Cell. Neurosci. 8.November (Nov. 2014). ISSN: 16625102. DOI: 10.3389/fncel.2014.00380.

[41] Francesca Salani et al. "Is Innate Memory a Double-Edge Sword in Alzheimer's Disease? A Reappraisal of New Concepts and Old Data". In: Front. Immunol. 10 (Aug. 2019), p. 1768. ISSN: 16643224. DOI: 10.3389/fimmu. 2019.01768. 
[42] Gilbert Di Paolo and Tae-Wan Kim. "Linking lipids to Alzheimer's disease: cholesterol and beyond". In: Nat. Rev. Neurosci. 12.5 (May 2011). ISSN: 1471003X. DOI: $10.1038 / \mathrm{nrn} 3012$.

[43] Hannah Chew, Victoria A. Solomon, and Alfred N. Fonteh. "Involvement of Lipids in Alzheimer's Disease Pathology and Potential Therapies". In: Front. Physiol. 11 (June 2020). ISSN: 1664-042X. DOI: 10.3389/fphys.2020.00598.

[44] Yu-Chia Kao et al. "Lipids and Alzheimer's Disease". In: Int. J. Mol. Sci. 21.4 (Feb. 2020). ISSN: 1422-0067. DOI: 10.3390/ijms21041505.

[45] Serena Sanna et al. "Common variants in the GDF5-UQCC region are associated with variation in human height". In: Nature Genetics 40.2 (2008), pp. 198-203. ISSN: 10614036 . DOI: $10.1038 / \mathrm{ng} \cdot 74$.

[46] Ulrike Künzel et al. "FRMD8 promotes inflammatory and growth factor signalling by stabilising the iRhom/ADAM17 sheddase complex". In: eLife 7 (2018). ISSN: 2050084X. DOI: 10.7554/eLife.35012.

[47] Damian Szklarczyk et al. "STRING v11: Protein-protein association networks with increased coverage, supporting functional discovery in genome-wide experimental datasets". In: Nucleic Acids Research 47.D1 (2019), pp. D607-D613. ISSN: 13624962. DOI: 10.1093/nar/gky1131.

[48] Hou Feng Zheng et al. "Whole-genome sequencing identifies EN1 as a determinant of bone density and fracture". In: Nature 526.7571 (Oct. 2015), pp. 112117. ISSN: 14764687. DOI: 10.1038/nature14878. URL: https://pubmed.ncbi. nlm.nih.gov/26367794/.

[49] Jonathan A Mitchell et al. "Rare EN1 Variants and Pediatric Bone Mass". In: J. Bone Miner. Res. 31.8 (Aug. 2016), pp. 1513-1517. ISSN: 15234681. DOI: 10 . 1002/jbmr.2833. URL: http://doi.wiley.com/10.1002/jbmr. 2833.

[50] Jian Zhou and Olga G. Troyanskaya. "Predicting effects of noncoding variants with deep learning-based sequence model". eng. In: Nat Methods 12.10 (Oct. 2015), pp. 931-934. ISSN: 1548-7105. DOI: 10.1038/nmeth. 3547.

[51] Alexis Battle et al. "Characterizing the genetic basis of transcriptome diversity through RNA-sequencing of 922 individuals". In: Genome Res. 24.1 (2014), pp. 14-24. ISSN: 10889051. DOI: $10.1101 / \mathrm{gr} .155192 .113$. arXiv: NIHMS150003.

[52] Michael Wainberg et al. "Opportunities and challenges for transcriptome-wide association studies." In: Nat Genet 51.4 (Mar. 2019), pp. 592-599. ISSN: 1061-

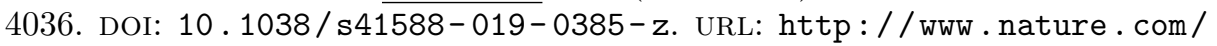
articles/s41588-019-0385-z (visited on 07/04/2019).

[53] GTEx Consortium et al. "Genetic effects on gene expression across human tissues." In: Nature 550.7675 (Oct. 2017), pp. 204-213. ISSN: 0028-0836. DOI: 10. 1038/nature24277. URL: http : / / www . nature . com/articles / nature24277 (visited on 01/10/2020). 
[54] Tuuli Lappalainen et al. "Transcriptome and genome sequencing uncovers functional variation in humans." In: Nature 501.7468 (Sept. 2013), pp. 506-511. DOI: 10. 1038 / nature12531. URL: http: / / dx . doi . org / 10 . 1038 / nature12531 (visited on 06/14/2019).

[55] Sharon R. Browning and Brian L. Browning. "Rapid and accurate haplotype phasing and missing-data inference for whole-genome association studies by use of localized haplotype clustering". In: Am. J. Hum. Genet. (2007). ISSN: 00029297. DOI: 10.1086/521987.

[56] Brian L. Browning, Ying Zhou, and Sharon R. Browning. "A One-Penny Imputed Genome from Next-Generation Reference Panels". In: Am. J. Hum. Genet. (2018). ISSN: 15376605. DOI: 10.1016/j.ajhg.2018.07.015.

[57] Christopher C. Chang et al. "Second-generation PLINK: Rising to the challenge of larger and richer datasets". In: Gigascience (2015). ISSN: 2047217X. DOI: 10. 1186/s13742-015-0047-8. arXiv: 1410.4803 .

[58] Elisa Mariella, Elena Grassi, and Paolo Provero. "eQTL Mapping Using Transcription Factor Affinity." In: Methods Mol Biol 2082 (2020), pp. 39-49. DOI: 10.1007/978-1-0716-0026-9_3. URL: http://dx.doi.org/10.1007/978-10716-0026-9_3 (visited on 12/07/2020).

[59] Adam Frankish et al. "GENCODE reference annotation for the human and mouse genomes". In: Nucleic Acids Res. (2019). ISSN: 13624962. DOI: 10.1093/ nar/gky955.

[60] Oliver Stegle et al. "Using probabilistic estimation of expression residuals (PEER) to obtain increased power and interpretability of gene expression analyses". In: Nat. Protoc. 7.3 (Mar. 2012), pp. 500-507. ISSN: 17542189. DOI: 10.1038/nprot. 2011.457. URL: https://github.com/PMBio/peer/.

[61] Ben Bolstad. "Probe Level Quantile Normalization of High Density Oligonucleotide Array Data". 2001. URL: http://bmbolstad.com/stuff/qnorm.pdf.

[62] T. Mark Beasley, Stephen Erickson, and David B. Allison. "Rank-based inverse normal transformations are increasingly used, but are they merited?" In: Behav. Genet. 39.5 (Sept. 2009), pp. 580-595. ISSN: 00018244. DOI: $10.1007 /$ s10519-009-9281-0. URL: /pmc/articles/PMC2921808/?report=abstract\% 20https://www.ncbi.nlm.nih.gov/pmc/articles/PMC2921808/.

[63] Ben Neale. UK Biobank - Neale lab. 2018. URL: http : / / www . nealelab . is / uk-biobank /\%20http : / / www . nealelab. is / uk-biobank (visited on $09 / 30 / 2020)$. 